Reconst ruct $i$ on of hi gh tempor al resol ut $i$ on Thomson scattering dat a during a modul ated el ect ron cycl ot ron resonance heat ing usi ng condi ti onal aver agi ng

\begin{tabular}{|l|l|}
\hline $\begin{array}{l}\text { j our nal or } \\
\text { publ i cat i on t i t l e }\end{array}$ & Revi ew of Sci ent i f i c I nst r ument s \\
\hline vol une & 87 \\
\hline number & 4 \\
\hline page $r$ ange & 043505 \\
\hline year & $2016-04$ 05 \\
\hline URL & ht t p: //hdl . handl e. net /10655/00012737 \\
\hline
\end{tabular}




\title{
Reconstruction of high temporal resolution Thom- son scattering data during a modulated electron cy- clotron resonance heating using conditional averaging
}

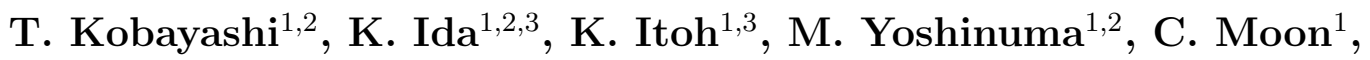 \\ S. Inagaki ${ }^{3,4}$, I. Yamada ${ }^{1}$, H. Funaba ${ }^{1}$, R. Yasuhara ${ }^{1}$, H. Tsuchiya ${ }^{1}$,

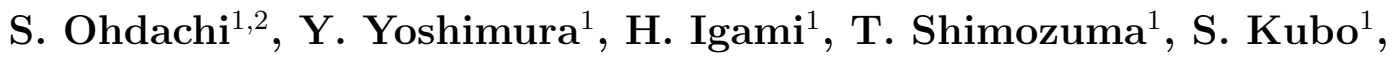 \\ and T. I. Tsujimura ${ }^{1}$ \\ 1 National Institute for Fusion Science, Toki 509-5292, Japan \\ 2 SOKENDAI (The Graduate University for Advanced Studies), Toki 509-5292, Japan \\ ${ }^{3}$ Research Center for Plasma Turbulence, Kyushu University, Kasuga 816-8580, Japan \\ ${ }^{4}$ Research Institute for Applied Mechanics, Kyushu University, Kasuga 816-8580, Japan \\ E-mail: kobayashi.tatsuya@LHD.nifs.ac.jp
}

\begin{abstract}
This paper provides a software application of the sampling scope concept for fusion research. The time evolution of Thomson scattering data is reconstructed with a high temporal resolution during a modulated electron cyclotron resonance heating (MECH) phase. The amplitude profile and the delay time profile of the heat pulse propagation are obtained from the reconstructed signal for discharges having on-axis and off-axis MECH depositions. The results are found to be consistent with the MECH deposition.
\end{abstract}

\section{Introduction}

Observation of dynamics is an excellent way for studying causal relation in physics. In fusion plasma experiments, for this purpose, modulation experiments have been widely 
performed. In particular, the electron cyclotron resonance heating (ECH) has been a useful tool for providing a modulation in the heat source, because the deposition can be localized, the deposition profile can be calculated relatively easily, and the modulation power and frequency can be precisely tuned. By observing the response to the modulated ECH $(\mathrm{MECH})$, a variety of modern physical issues, such as inward heat pinch $[1,2]$, non-diffusive transport $[3,4,5]$, magnetic field topology $[6,7]$, and turbulence response [8], have been investigated. In order to diagnose the fast response of the electron temperature to the $\mathrm{MECH}$, the electron cyclotron emission (ECE) measurement [9] is routinely used. Although the ECE measurement is powerful for the study of the plasma dynamics, the use can be restricted in a range of experimental conditions such as the electron density and the magnetic field. Moreover, it is known that the ECE signal suffers from non-thermal emission, which provides a temperature evaluation error under a condition such as low density and high ECH power input [10]. Not only for conducting a cross-check of results obtained with the ECE measurement but also for exploring the plasma dynamics in a parameter range beyond the ECE coverage, an alternative way to diagnose the electron temperature profile with a high temporal resolution is desired. A possible alternative can be the Thomson scattering system, but the temporal resolution of the Thomson scattering system is far poorer than the ECE measurement in general.

In this paper, we propose a new method to reconstruct the time evolution of Thomson scattering data with a high temporal resolution during a MECH phase. The method is a software application of the sampling scope concept to the Thomson scattering 
data. (For an application of the hardware sampling scope for plasma diagnostics, see, e.g., [11]. ) Fourier amplitude and delay time profiles of the heat pulse propagation are obtained from the reconstructed signal for discharges having on-axis and off-axis MECH depositions.

\section{Experimental setup}

The experiments were conducted in the Large Helical Device (LHD). The LHD is a Heliotron device typically having a major radius of $R_{\mathrm{ax}}=3.5-4.1 \mathrm{~m}$ and an averaged minor radius of $a \sim 0.6 \mathrm{~m}$. The major radius can be controlled externally in order to alter the plasma regime, such as MHD instability or energetic particle confinement. Since the magnetic surface in the LHD is generated not by the plasma current but by the external helical coils, current driven MHD instabilities are rarely excited. This property is desirable for the perturbative experiments such as the heat/cold pulse propagation experiment $[4,5,6,12]$. The Thomson scattering system diagnoses the electron temperature and density profiles with more than 140 spatial points [13]. The YAG laser of the Thomson scattering system has a repetition frequency of $f_{\mathrm{TS}}=30 \mathrm{~Hz}$, and a pulse width of 10 ns. 


\section{Reconstruction of Thomson scattering data with a high temporal resolution}

The Thomson scattering data is reconstructed using the conditional averaging technique.

If one takes the MECH frequency $f_{\mathrm{MECH}}$ and the Thomson laser repetition frequency $f_{\text {TS }}$ as numbers that are not in the multiple relation, the Thomson scattering system diagnoses various phases in the MECH period as time passes. By taking the measured electron temperature as a function of the measured phase, the time sequence within one period of the MECH can be reconstructed. The time period that is needed to complete a revolution of the measurement phase is given as $T=\left[\text { G.C.D. }\left(f_{\mathrm{MECH}}, f_{\mathrm{TS}}\right)\right]^{-1}$ sec., where G.C.D. is the greatest common divisor for frequencies given by integers. As a result, one period of the perturbation is reconstructed with $N \equiv T f_{\text {TS }}$ points. Note that the number $N$ takes a larger value if one chooses $f_{\mathrm{MECH}}$ and $f_{\mathrm{TS}}$ as coprime numbers than in the other case. Therefore, the equivalent sampling rate of the reconstructed signal is given as $N f_{\mathrm{MECH}}=f_{\mathrm{MECH}} f_{\mathrm{TS}}\left[\text { G.C.D. }\left(f_{\mathrm{MECH}}, f_{\mathrm{TS}}\right)\right]^{-1} \mathrm{~Hz}$.

\subsection{Comparison between the conditional averaged ECE data and the reconstructed}

\section{Thomson scattering data}

The first example of the reconstruction is shown for a discharge having the magnetic configuration with the vacuum magnetic axis position of $R_{\mathrm{ax}}=3.6 \mathrm{~m}$ and the magnetic field strength on the magnetic axis of $B_{\mathrm{ax}}=2.75 \mathrm{~T}$. A typical time evolution of the discharge is shown in Fig. 1. The plasma is mainly sustained with two neutral beams 
(NBs) injected in the co- and counter-directions with respect to the direction of the toroidal magnetic field. The power of the NBs is $P_{\mathrm{NB}}=1.5 \mathrm{MW}$ each, so that the beam driven current is canceled. The analysis is performed in $1.7 \mathrm{sec}$. of the stationary period, where the line averaged density and the plasma stored energy is almost constant [Fig. 1 (a)]. Three systems of the MECH with a modulation frequency of $f_{\mathrm{MECH}}=25 \mathrm{~Hz}$ are used for applying the perturbation in the electron temperature profile [Fig. 1 (b)]. The MECH power is deposited mainly in the core region, $r_{\text {eff }} / a_{99}<0.2$, where $r_{\text {eff }}$ and $a_{99}$ show the averaged minor radius on a magnetic flux surface and the effective minor radius in which $99 \%$ of the plasma kinetic energy is confined, respectively. The repetition frequency of the Thomson scattering system is $f_{\mathrm{TS}}=30 \mathrm{~Hz}$. Figure 1 (b) shows the time evolution of the electron temperature at the core code and the midradius, measured with the Thomson scattering and the ECE. The Thomson scattering signals are spatially averaged using 5 or 6 points in order to reduce the scatter of the data points. The Thomson electron temperature points overlap the ECE signal most of the time, although the DC value of the ECE is relatively larger. The waveform of the ECE signal in each MECH cycle shows a good reproducibility, which is a premise of the method. Figure 1 (c) shows the time evolution of the measurement phase in the MECH period defined as $\Delta t\left(t_{\mathrm{TS}, j}\right)=\min \left[\tau_{i}-t_{\mathrm{TS}, j}\right]$, where $\tau_{i}$ and $t_{\mathrm{TS}, j}$ are the $i$-th MECH turn-on time and the $j$-th Thomson laser injection time, respectively. For the present case, the G.C.D. between $f_{\mathrm{MECH}}$ and $f_{\mathrm{TS}}$ is five so that $T=0.2 \mathrm{sec}$. and $N=6$. The Thomson electron temperature oscillates with the period of $T=0.2 \mathrm{sec}$, following 
the revolution of the measurement period.

By taking the measured electron temperature perturbation $\delta T_{\mathrm{e}}$ as a function of the measured phase $\Delta t$, the time sequence within one period of the MECH can be reconstructed as shown in Fig. 2. The definition of the temperature perturbation is given as $\delta T_{\mathrm{e}} \equiv T_{\mathrm{e}}-\left\langle T_{\mathrm{e}}\right\rangle$, where $\left\langle T_{\mathrm{e}}\right\rangle$ is the time averaged electron temperature. The reconstructed Thomson data points (circles and diamonds) agree well with the conditional averaged ECE signals, although there is a scatter of the points in the Thomson electron temperature. The scatter of the points is found to shape the Gaussian distribution so that accumulation of the points using a long pulse of the MECH benefits to reduce uncertainty.

\subsection{Reconstruction of Thomson scattering data in low density discharges}

Now the method is applied to the other discharges, which have a low line averaged density of $\left\langle n_{\mathrm{e}}\right\rangle \sim 0.4 \times 10^{19} \mathrm{~m}^{-3}$ and a high $\mathrm{ECH}$ power of $P_{\mathrm{ECH}}=3 \mathrm{MW}$. In these cases, the discharge is subject to the non-thermal electrons that provide a serious error to the evaluated temperature [10]. The vacuum magnetic axis position and the magnetic field strength is $R_{\mathrm{ax}}=3.53 \mathrm{~m}$ and $B_{\mathrm{ax}}=2.8 \mathrm{~T}$, respectively. The plasma is sustained with two NBs whose total power is $P_{\mathrm{NB}}=5 \mathrm{MW}$, and $P_{\mathrm{ECH}}=2.3 \mathrm{MW}$ of the MECH with the frequency of $f_{\mathrm{MECH}}=19 \mathrm{~Hz}$ is applied. Since $f_{\mathrm{TS}}=30 \mathrm{~Hz}, f_{\mathrm{MECH}}$ and $f_{\mathrm{TS}}$ are coprime so that $T=1 \mathrm{sec}$. and $N=f_{\mathrm{TS}}=30$. The equivalent sampling rate for one revolution is $N f_{\mathrm{MECH}}=570 \mathrm{~Hz}$, which is 19 times larger than the original Thomson laser 
repetition frequency. Figure 3 shows the reconstructed Thomson signals at $r_{\text {eff }} / a_{99} \sim 0$ and $r_{\text {eff }} / a_{99} \sim 0.45$. Data points outside the center period of the $\mathrm{MECH}(|\Delta t|>26.3 \mathrm{~ms})$ are repeats of the reconstructed data points in the center period $(|\Delta t|<26.3 \mathrm{~ms})$. The points are homogeneously distributed in the center period of the MECH. Comparing two reconstructed signals at the core and the mid-radius, the difference in the waveform is clearly seen. At the plasma core, a rapid response of the electron temperature to the $\mathrm{MECH}$ is seen, whereas the response is relatively slow at the mid radius. In addition, the phase difference between them can also be detected.

The heat pulse propagation is analyzed based on the reconstructed Thomson scattering data. Here, two discharges having almost the identical profiles and time evolutions but different MECH depositions are used. Figure 4 shows the radial profile of the electron density, the electron temperature, the perturbation amplitude, and the delay time of the heat pulse for the on-axis MECH discharge (left column) and the offaxis MECH discharge (right column). The perturbation amplitude and the delay time is calculated for the fundamental mode by the Fourier transform of the reconstructed signal. For Figs. 4 (a), (b), (e), and (f), the time average is performed for the second half of the MECH-on phase $\left(0.25<\tau f_{\mathrm{MECH}}<0.5\right)$ and for the second half of the MECH-off phase $\left(-0.25<\tau f_{\mathrm{MECH}}<0\right)$. The evaluated MECH deposition profiles using the ray-tracing code LHDGauss [14] are plotted in (b) and (f). The change in the electron density profile is much smaller than that in the electron temperature profile in both discharges. For the case of the on-axis MECH, the electron temperature shows the 
central peaked profile, while there is the flattening region in the center $\left|r_{\text {eff }} / a_{99}\right|<0.5$ for the off-axis MECH case. As shown in Figs. 4 (c) and (d), the central peaked perturbation amplitude profile and the monotonically increasing delay time profile are given for the on-axis MECH case. In contrast, the off-axis MECH deposition provides the flat amplitude and delay time profiles as shown in Figs. 4 (g) and (h). Note that the flat amplitude profile and the flat delay time profile in the off-axis case might be caused by either a relatively wider MECH deposition or a core stochastization of the magnetic field. The results of the Fourier analysis are found to be consistent with the MECH deposition.

\section{Summary}

In this paper, we proposed a new method to reconstruct the time evolution of a Thomson scattering data with a high temporal resolution during a MECH phase. The method is a software application of the sampling scope concept to the Thomson scattering data. By choosing the proper MECH frequency $f_{\mathrm{MECH}}$ and Thomson scattering laser repetition frequency $f_{\mathrm{TS}}$, one period of the modulation electron temperature profile is reconstructed. The equivalent sampling rate of the reconstructed signal is given as $f_{\mathrm{MECH}} f_{\mathrm{TS}}\left[\text { G.C.D. }\left(f_{\mathrm{MECH}}, f_{\mathrm{TS}}\right)\right]^{-1} \mathrm{~Hz}$, where G.C.D. is the greatest common divisor for the frequencies given by integers. Therefore, the equivalent sampling rate can be large if one takes $f_{\mathrm{MECH}}$ and $f_{\mathrm{TS}}$ as coprime numbers. The amplitude profile and the delay time profile of the heat pulse propagation were obtained from the reconstructed signal 
for discharges having on-axis and off-axis MECH depositions, and the results were found to be consistent with the MECH deposition.

Using this method, plasma dynamics can be analyzed in a parameter range outside the ECE coverage. In the case of the LHD, the number of the spatial points of the Thomson scattering system well exceeds that of the ECE, covering a wide radial range in both the high-field-side and the low-field-side of the plasma. We believe that combining the reconstructed Thomson scattering signal to the conventional ECE signal, a deeper understanding of plasma physics, in particular that concerning the energy transport, can be achieved in the future.

In general, this method is applicable not only for the $\mathrm{MECH}$ experiments but also for other repetitive plasma dynamics, e.g., edge localized modes or limit cycle oscillations, in cases in which a trigger for the events can be explicitly defined. Moreover, the Thomson scattering system can be replaced with other diagnostics, such as the charge exchange spectroscopy or the impurity spectrum measurements, for measurements of other plasma parameters.

\section{Acknowledgements}

The authors acknowledge all the members of the LHD Experiment Group for their assistance. The authors also thank Profs. S. Sakakibara, S.-I. Itoh, and A. Fujisawa for strong support, and Y. Nagayama and K. Tanaka for useful discussions. This work is partly supported by the National Institute for Fusion Science grant administrative 
budget (ULHH033) and the Grant-in-Aid for Scientific Research of JSPS (15H02336, 23244113, 26887047, and 15H02155). 


\section{Reference}

[1] P Mantica, G Gorini, G M D Hogeweij, N J Lopes Cardozo, and A M R Schilham 2000 Phys. Rev. Lett. 854534

[2] P Mantica, F Ryter, C Capuano, H U Fahrbach, F Leuterer, W Suttrop, Jan Weiland, and ASDEX-Upgrade Team 2006 Plasma Phys. Control. Fusion 48385

[3] K W Gentle, M E Austin, J C DeBoo, T C Luce, and C C Petty 2006 Phys. Plasmas 13012311

[4] S Inagaki, T Tokuzawa, N Tamura, S-I Itoh, T Kobayashi, K Ida, T Shimozuma, S Kubo, K Tanaka, T Ido, et al 2013 Nucl. Fusion 53113006

[5] S Inagaki, T Tokuzawa, T Kobayashi, S-I Itoh, K Itoh, K Ida, S Kubo, T Shimozuma, N Tamura, A Fujisawa, et al 2013 Plasma Fusion Res. 81202172

[6] K Ida, M Yoshinuma, H Tsuchiya, T Kobayashi, C Suzuki, M Yokoyama, A Shimizu, K Nagaoka, S Inagaki, K Itoh, et al 2015 Nat. Commun. 65816

[7] K Ida, T Kobayashi, T E Evans, S Inagaki, M E Austin, M W Shafer, S Ohdachi, Y Suzuki, S-I Itoh, and K Itoh 2015 Sci. Rep. 516165

[8] J C DeBoo, C Holland, T L Rhodes, L Schmitz, G Wang, A E White, M E Austin, E J Doyle, J Hillesheim, W A Peebles, et al 2010 Phys. Plasmas 17056105

[9] K Kawahata, Y Nagayama, S Inagaki, Y Ito, et al 2003 Rev. Sci. Instrum. 74 1449-1452

[10] S Kubo, H Igami, Y Nagayama, S Muto, T Shimozuma, Y Yoshimura, H Takahashi, and T Notake 2009 J. Plasma Fusion Res. SERIES 81095

[11] Y Kogi, K Uchida, A Mase, L Bruskin, M Ignatenko, T Tokuzawa, Y Nagayama, and K Kawahata 2004 Rev. Sci. Instrum. $\mathbf{7 5} 3837$

[12] N Tamura, S Inagaki, K Tanaka, C Michael, T Tokuzawa, T Shimozuma, S Kubo, R Sakamoto, K Ida, K Itoh, et al 2007 Nucl. Fusion 47449

[13] I Yamada, K Narihara, H Funaba, T Minami, H Hayashi, T Kohmoto, LHD Experiment Group, et al 2010 Fusion Sci. Technol. 58 345-351 
[14] T Ii Tsujimura, S Kubo, H Takahashi, R Makino, R Seki, Y Yoshimura, H Igami, T Shimozuma, K Ida, C Suzuki, et al 2015 Nucl. Fusion 55123019 


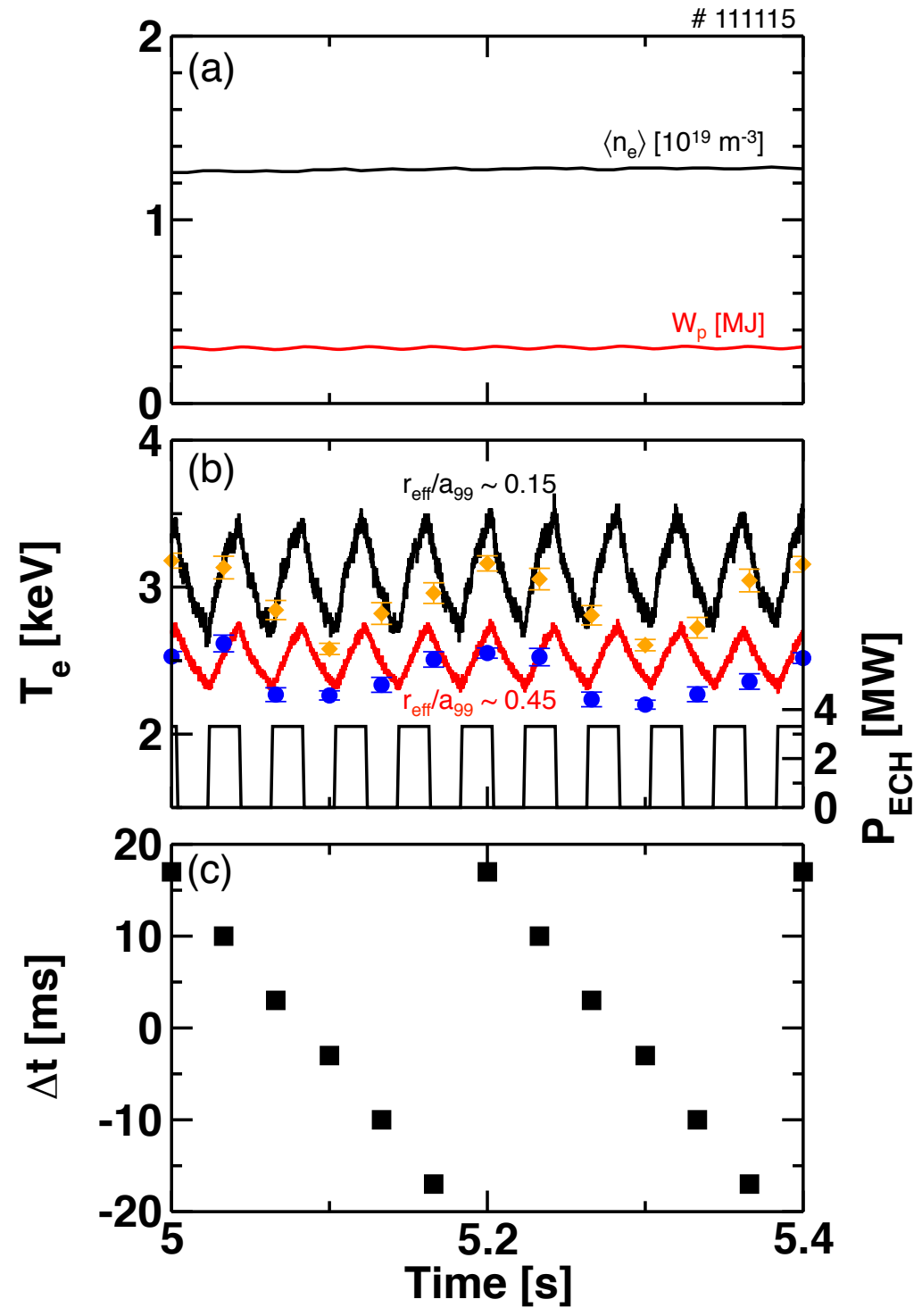

Figure 1. Time evolutions of (a) the line averaged density and the plasma stored energy, (b) the electron temperature measured with the ECE (black and red lines) and with the Thomson scattering system (circles and diamonds) and the ECH power, and (c) the phase of the Thomson laser injection in the MECH period. 


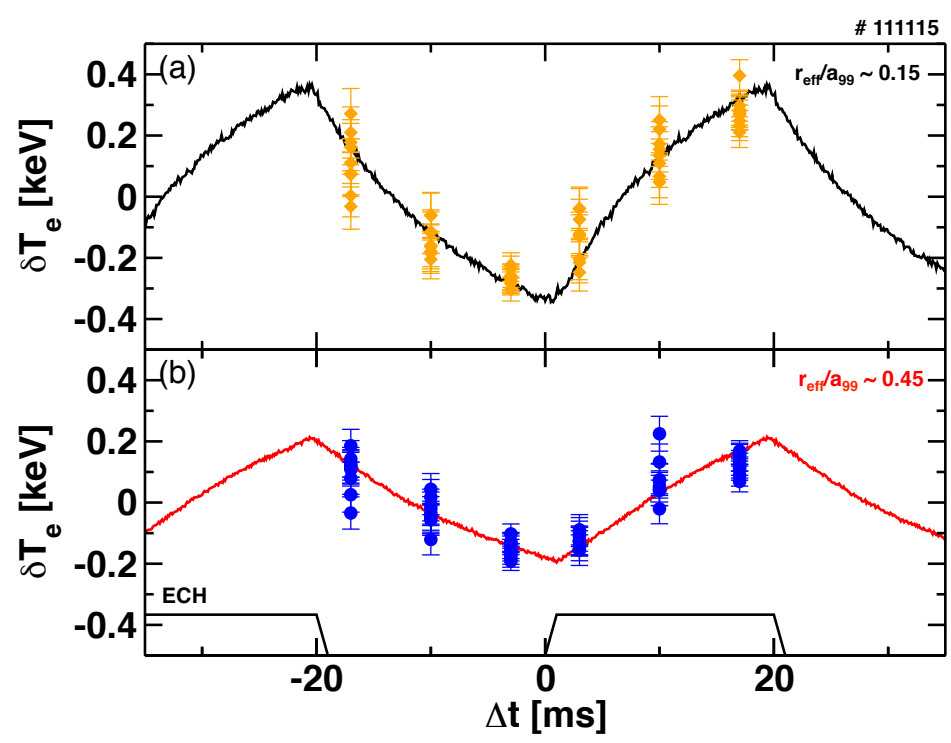

Figure 2. Time evolutions of the conditional averaged electron temperature measured with the ECE (black and red lines) and with the Thomson scattering system (circles and diamonds) at (a) $r_{\text {eff }} / a_{99} \sim 0.15$ and at (b) $r_{\text {eff }} / a_{99} \sim 0.45$. The Thomson scattering signals are spatially averaged using 5 or 6 points.

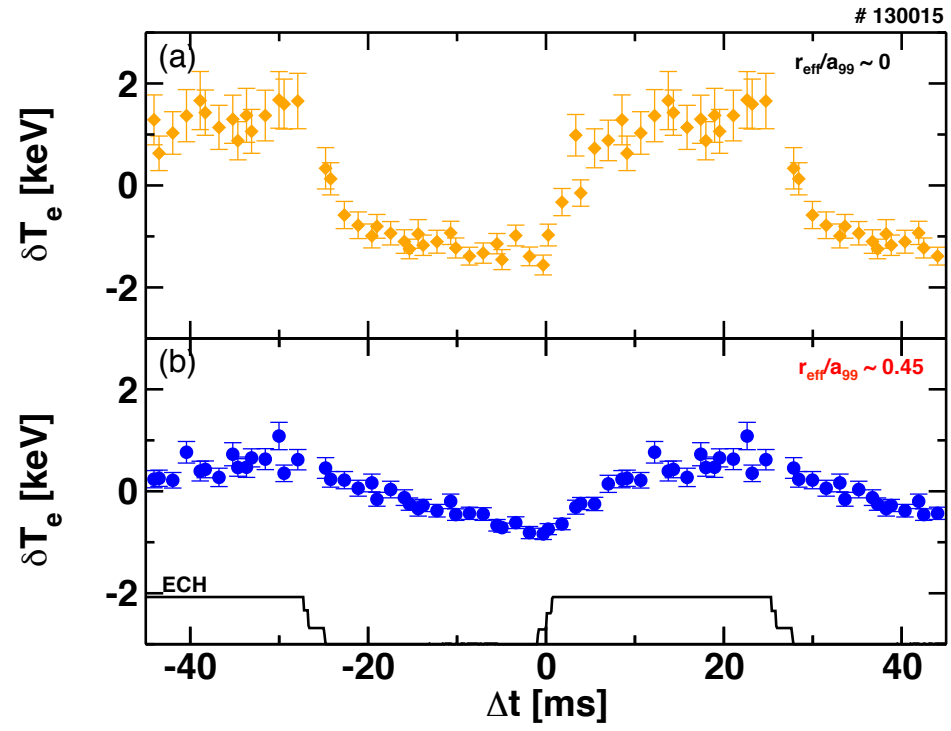

Figure 3. Time evolutions of the conditional averaged electron temperature measured with the Thomson scattering system at (a) $r_{\text {eff }} / a_{99} \sim 0$ and at (b) $r_{\text {eff }} / a_{99} \sim 0.45$. The Thomson scattering signals are spatially averaged using 5 or 6 points. 

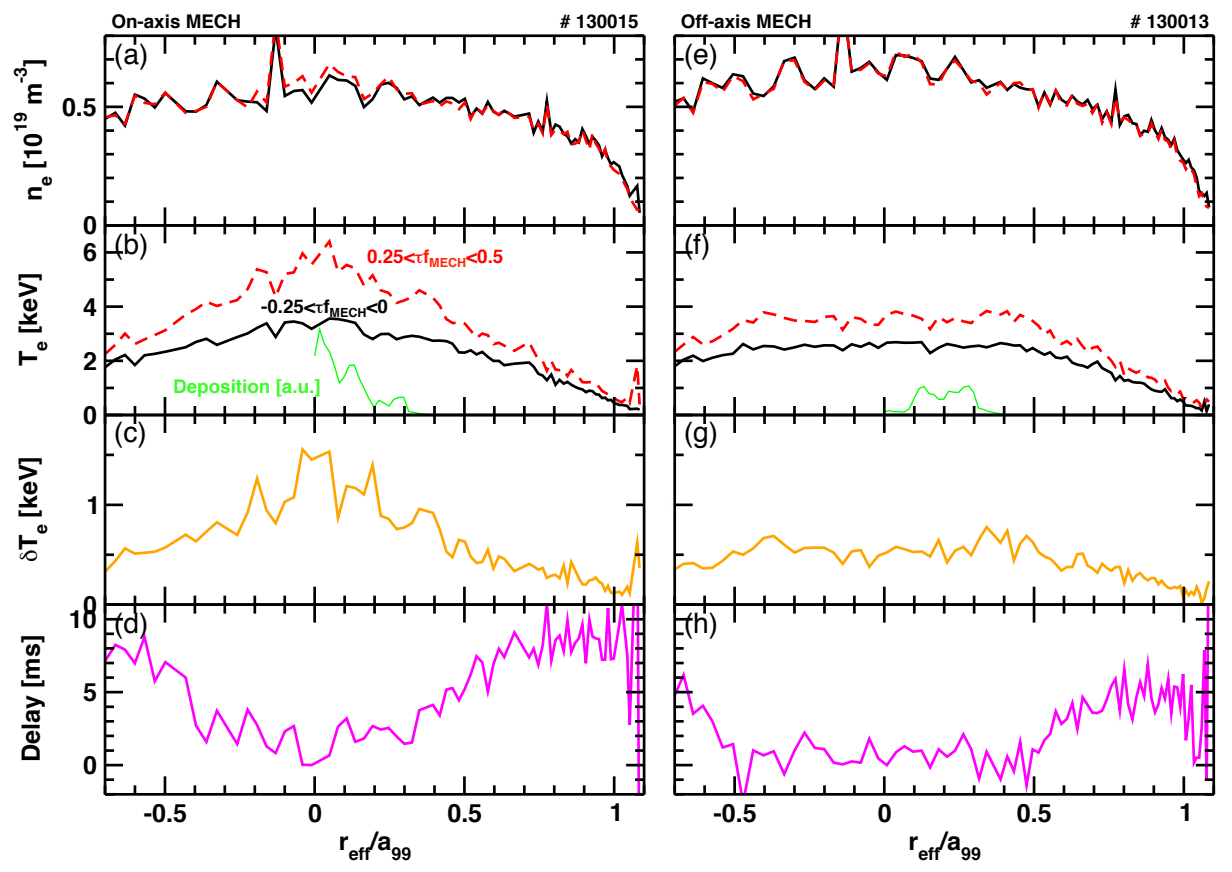

Figure 4. (left column) Radial profiles of (a) the conditional averaged electron density, (b) the conditional averaged electron temperature, (c) the perturbation amplitude, and (d) the delay time of the heat pulse for the discharge with the on-axis MECH (\#130015). (right column) (e)-(h) Those for the discharge with the off-axis MECH (\#130013). For the panels (a), (b), (f), and (g), the time average is performed for the second half of the MECH-on phase $\left(0.25<\tau f_{\mathrm{MECH}}<0.5\right.$, red) and for the second half of the MECH-off phase $\left(-0.25<\tau f_{\mathrm{MECH}}<0\right.$, black). 